(1)

CrossMark

\title{
Centrally located lung cancer and risk of occult nodal disease: an objective evaluation of multiple definitions of tumour centrality with dedicated imaging software
}

\author{
Roberto F. Casal ${ }^{1}$, Boris Sepesi ${ }^{2}$, Ala-Eddin S. Sagar ${ }^{1}$, Juerg Tschirren ${ }^{3}$, \\ Minxing Chen ${ }^{4}$, Liang Li ${ }^{4}$, Jennifer Sunny ${ }^{5}$, Joyce Williams ${ }^{1}$, Horiana B. Grosu ${ }^{1}$, \\ George A. Eapen ${ }^{1}$, Carlos A. Jimenez ${ }^{1}$ and David E. Ost ${ }^{1}$
}

Affiliations: ${ }^{1}$ Dept of Pulmonary Medicine, The University of Texas MD Anderson Cancer Center, Houston, TX, USA. ${ }^{2}$ Dept of Cardiothoracic Surgery, The University of Texas MD Anderson Cancer Center, Houston, TX, USA. ${ }^{3}$ Engineering Dept, VIDA Diagnostics, Coralville, IA, USA. ${ }^{4}$ Dept of Biostatistics, The University of Texas MD Anderson Cancer Center, Houston, TX, USA. ${ }^{5}$ Dept of Medicine, The University of Texas Health Science Center at Houston, Houston, TX, USA.

Correspondence: Roberto F. Casal, Dept of Pulmonary Medicine, The University of Texas MD Anderson Cancer Center, 1400 Pressler Street, Unit 1462, Houston, TX 77030, USA. E-mail: rfcasalamdanderson.org

@ERSpublications

In T1N0M0 nonsmall cell lung cancer, the prevalence of occult mediastinal disease is low regardless of tumour location. However, centrally located tumours are at risk of upstaging to any N. Here, two specific definitions of central tumours are identified. http://ow.ly/pqa130nIokW

Cite this article as: Casal RF, Sepesi B, Sagar A-ES, et al. Centrally located lung cancer and risk of occult nodal disease: an objective evaluation of multiple definitions of tumour centrality with dedicated imaging software. Eur Respir J 2019; 53: 1802220 [https://doi.org/10.1183/13993003.02220-2018].

\section{ABSTRACT}

Introduction: Current guidelines recommend invasive mediastinal staging in patients with centrally located radiographic stage T1N0M0 nonsmall cell lung cancer (NSCLC). The lack of a specific definition of a central tumour has resulted in discrepancies among guidelines and heterogeneity in practice patterns. Methods: Our objective was to study specific definitions of tumour centrality and their association with occult nodal disease. Pre-operative chest computed tomography scans from patients with clinical (c) T1N0M0 NSCLC were processed with a dedicated software system that divides the lungs in thirds following vertical and concentric lines. This software accurately assigns tumours to a specific third based both on the location of the centre of the tumour and its most medial aspect, creating eight possible definitions of central tumours.

Results: 607 patients were included in our study. Surgery was performed for 596 tumours (98\%). The overall pathological (p) N disease was: 504 (83\%) N0, $56(9 \%)$ N1, 47 (8\%) N2 and no N3. The prevalence of N2 disease remained relatively low regardless of tumour location. Central tumours were associated with upstaging from $\mathrm{cN} 0$ to any $\mathrm{N}(\mathrm{pN} 1 / \mathrm{pN} 2)$. Two definitions were associated with upstaging to any $\mathrm{N}$ : concentric lines, inner one-third, centre of the tumour (OR 3.91, 95\% CI 1.85-8.26; p<0.001) and concentric lines, inner two-thirds, most medial aspect of the tumour (OR 1.91, 95\% CI 1.23-2.97; p=0.004).

Conclusions: We objectively identified two specific definitions of central tumours. While the rate of occult mediastinal disease was relatively low regardless of tumour location, central tumours were associated with upstaging from cN0 to any $\mathrm{N}$.

This article has supplementary material available from erj.ersjournals.com

Received: Nov 212018 | Accepted after revision: Feb 082019

Copyright OERS 2019 


\section{Introduction}

Accurate mediastinal staging is crucial in the management of patients with lung cancer. It defines prognosis, dictates treatment and it allows for meaningful comparisons among different therapeutic strategies in clinical trials. Techniques for mediastinal staging can be divided in two broad categories: noninvasive (radiographic) and invasive. In the context of a radiographically normal mediastinum, current guidelines suggest that invasive mediastinal staging should be performed for patients with radiographic N1 disease, tumours $>3 \mathrm{~cm}$ and for patients with "centrally located" T1N0M0 lung cancer [1-3]. Unfortunately, the definition of "centrally located" tumours for this latter group is not clear. While the American College of Chest Physicians guidelines define central tumours as those located within the inner "one-third" of the lung, the National Comprehensive Cancer Network and the European Society of Thoracic Surgery guidelines define central tumours as those within the inner "two-thirds" of the lung [1-3]. Data to support one definition or the other are scant and inconsistent [4-10]. In addition, methods to delineate the three thirds of the lung or to classify a tumour that crosses a boundary (i.e. between a third that is considered central and one that is considered peripheral) have not been defined. Most studies evaluating tumour centrality informing these guidelines were retrospective in nature, with nonspecific definitions and with inherent subjectivity given by the individuals assessing images to classify tumours as central or peripheral [4-10]. A recent online survey performed by our group demonstrated heterogeneous practice patterns arising from the lack of a uniform, clear, objective and evidence-based definition of central tumours [11].

The purpose of this study is to objectively evaluate different definitions of tumour centrality for patients with clinical (c) T1N0M0 tumours and their association with occult nodal disease in order to better inform future lung cancer practice guidelines.

\section{Methods \\ The study was conducted at The University of Texas MD Anderson Cancer Center (Houston, TX, USA) where pertinent institutional review board approval was obtained (approval PA16-1061). Prospectively collected databases of thoracic surgical cases and cytology samples of endobronchial ultrasound transbronchial needle aspiration (EBUS-TBNA) were queried between January 1, 2009 and December 31, 2016. Patients with radiographic T1N0M0 NSCLC who underwent surgical resection or who underwent EBUS-TBNA that upstaged them to N2/N3 disease preventing upfront surgical resection were included in this study. The eighth edition of the TNM (tumour, node, metastasis) classification was utilised, with T1 defined as tumours of up to $3 \mathrm{~cm}$ in the longest dimension [12]. Radiographic N0 was defined as both mediastinal and hilar lymph nodes of up to $1 \mathrm{~cm}$ in the short axis by computed tomography (CT) and standardised uptake values $<2.5$ by positron emission tomography (PET) or PET-CT. Only patients with guideline-consistent nodal dissection during surgery were included [2]. The prevalence of nodal disease was determined by the pathology results of nodal dissection or by the positive results of EBUS-TBNA (when N2/N3 disease was found, preventing surgical resection). EBUS-TBNA was performed in N3-N2- $\mathrm{N} 1$ fashion with onsite cytology examination, sampling all lymph nodes of $\geqslant 5 \mathrm{~mm}$ in the short axis.}

A dedicated imaging software system (VIDA Lung Zones; VIDA Diagnostics, Coralville, IA, USA) was designed for this study (see further details in the supplementary material). The software automatically divides each lung into thirds (inner, middle and outer) following two patterns: "vertical" and "concentric". The "vertical" pattern consists of straight lines that divide the lung in the sagittal plane, while the "concentric" pattern consists of lines that follow the contour of the lung (figure 1). Next, the operator needs to manually select both the most medial (closer to the hilum) aspect of the tumour and the tumour centre in order to assign it to a specific third (in cases of semisolid tumours, the solid component was utilised). This is performed while viewing simultaneously axial, coronal and sagittal CT cuts (supplementary material). This last feature (selecting the centre and the medial aspect of the tumour) was designed to solve the problem of tumours that cross a boundary (a line dividing two thirds), raising the question of which third they belong to (most medial one or the third where the centre of the tumour is located in). Based on the aforementioned, eight definitions of central tumours were created (table 1). Pre-operative chest CT scans from all patients were processed with our dedicated software. The operators who processed the scans were "blind" to pathology results. The task of these operators was to identify the nodule in the CT scan, and mark both the centre of the tumour and its most medial aspect. The software would then automatically assign the tumour to a specific third following the vertical and concentric patterns, and utilising both the most medial aspect of the tumour and the centre of the tumour as described earlier.

The primary objective was to evaluate the association between each of the different definitions of central tumours (table 1) and the presence of occult nodal disease. Nodal disease was analysed as mediastinal disease $(\mathrm{N} 2 / \mathrm{N} 3)$ or "any" nodal disease $(\mathrm{N} 1 / \mathrm{N} 2 / \mathrm{N} 3)$. The highest $\mathrm{N}$ stage was utilised for each patient. 



FIGURE 1 Processing of chest computed tomography with dedicated image software. Notice how the same right upper lobe nodule (cross-lines) can be assigned to the middle third by the "vertical" line pattern and to the outer third by the "concentric" line pattern.

TABLE 1 Definitions of central tumours

\begin{tabular}{lccc} 
Definition & Line pattern & Third & Tumour aspect \\
\hline $\mathbf{1}$ & Concentric & Inner one-third & Medial \\
$\mathbf{2}$ & Concentric & Inner two-thirds & Medial \\
$\mathbf{3}$ & Concentric & Inner one-third & Centre \\
$\mathbf{4}$ & Concentric & Inner two-thirds & Centre \\
$\mathbf{5}$ & Vertical & Inner one-third & Medial \\
$\mathbf{6}$ & Vertical & Inner two-thirds & Medial \\
$\mathbf{7}$ & Vertical & Inner one-third & Centre \\
$\mathbf{8}$ & Vertical & Inner two-thirds & Centre
\end{tabular}


Univariate logistic regression was applied to predict the odds of nodal disease by using each of the eight definitions of tumour centrality. Odds ratios and 95\% confidence intervals were reported from binary regression models for both mediastinal and any nodal disease. Multivariate analysis was run for the definitions that achieved statistical significance (at $\alpha=0.05$ ) in univariate analysis controlling for multiple covariates: tumour histology, tumour differentiation, anatomical tumour location, radiographic $\mathrm{T}$ descriptor, nodule type (solid, semisolid, ground glass) and fluorodeoxyglucose avidity. As part of a secondary analysis, the same process was repeated after excluding patients with ground-glass and carcinoid tumours (due to the expected low prevalence of occult nodal disease) and after excluding patients who did not have PET or PET-CT (since PET-CT is currently the standard of care for staging). When more than one definition was found to be associated with the outcome, the strength of association among the different definitions and outcome was compared utilising the difference in log odds ratios between the definitions calculated with $95 \%$ confidence intervals from nonparametric bootstrap with the use of Monte Carlo sampling. Analysis was performed with Stata version 15 (StataCorp, College Station, TX, USA).

\section{Results}

A total of 607 cT1N0M0 patients were included in our study and their pre-operative chest CT scans were analysed with our dedicated software. Surgery was performed for 596 tumours (98\%). EBUS-TBNA for nodal staging was performed in 121 patients $(20 \%)$ with only 11 tumours being upstaged to N2 disease and thereby preventing surgery. Pre-operative PET or PET-CT was available in 481 patients $(80 \%)$. The overall pathological (p) N disease was 504 (83\%) N0, $56(9 \%)$ N1, 47 (8\%) N2 and no N3. Baseline characteristics of patients and tumours are given in table 2.

\section{All patients}

Univariate analysis did not demonstrate a firm relationship between any of the proposed eight definitions of tumour centrality and the presence of occult $\mathrm{pN} 2 / \mathrm{pN} 3$ disease (table 3). There was a relationship between Definition 2 ("concentric" lines, inner two-thirds, medial aspect of the tumour) and occult pN2/ $\mathrm{pN} 3$ disease with an OR of 1.88 (95\% CI 1.00-3.51; $\mathrm{p}=0.049)$, but this did not reach statistical significance in multivariate analysis. Central tumour location was, however, associated with upstaging to any $\mathrm{N}$ (from $\mathrm{cN} 0$ to $\mathrm{pN} 1 / \mathrm{pN} 2 / \mathrm{pN} 3$ ). The definitions with the strongest relationship were Definition 2 ("concentric" lines, inner two-thirds, medial aspect of the tumour) and Definition 3 ("concentric" lines, inner one-third, centre of the tumour) (table 4). Both of them were tested in multivariate analysis against multiple confounders and they remained statistically significant (supplementary table E1). The difference in log OR between Definitions 3 and 1 was 0.679 (95\% CI $0.218-1.141$ ) indicating that Definition 3 is significantly better than Definition 1 in terms of its association with the outcome (upstage to pN1/pN2/pN3). However, we failed to demonstrate a difference between Definitions 2 and 3.

\section{Excluding patients without pre-operative staging PET-CT}

Univariate analysis did not demonstrate a firm relationship between any of the proposed eight definitions of tumour centrality and the presence of occult $\mathrm{pN} 2 / \mathrm{pN} 3$ disease (table 5 ). Central tumour location was associated with upstaging to any $\mathrm{N}$ (from $\mathrm{cN} 0$ to $\mathrm{pN} 1 / \mathrm{pN} 2 / \mathrm{pN} 3$ ). The definitions with the strongest relationship were Definition 2 ("concentric" lines, inner two-thirds, medial aspect of the tumour) and Definition 3 ("concentric" lines, inner one-third, centre of the tumour) (table 6). Both definitions was tested in multivariate analysis and they remained statistically significant (supplementary table E2). We failed to demonstrate a difference in log odds ratio between Definitions 2 and 3.

\section{Excluding patients with ground-glass opacities and patients with carcinoid tumours}

Univariate analysis demonstrated relationship between Definition 2 ("concentric" lines, inner two-thirds, medial aspect of the tumour) and occult pN2/pN3 disease with an OR of 1.95 (95\% CI 1.03-3.71; $\mathrm{p}=0.041$ ), but this did not reach statistical significance in multivariate analysis (supplementary table E3). Central tumour location was associated with upstaging to any $\mathrm{N}$ (from cN0 to pN1/pN2/pN3). Definitions 1, 2 and 3 were associated with this outcome in univariate analysis (table 7), but only Definitions 2 and 3 remained statistically significant in multivariate analysis (supplementary table E4). Again, we failed to demonstrate a difference in log odds ratio between Definitions 2 and 3.

\section{Excluding patients with ground-glass opacities, carcinoid tumours and those without pre-operative staging PET-CT}

Univariate analysis did not demonstrate a firm relationship between any of the proposed eight definitions of tumour centrality and the presence of occult $\mathrm{pN} 2 / \mathrm{pN} 3$ disease (supplementary table E5). Central tumour location was associated with upstaging to any $\mathrm{N}$ (from $\mathrm{cN} 0$ to $\mathrm{pN} 1 / \mathrm{pN} 2 / \mathrm{pN} 3$ ). The definitions with the strongest relationship were Definition 2 ("concentric" lines, inner two-thirds, medial aspect of the tumour) and Definition 3 ("concentric" lines, inner one-third, centre of the tumour) (supplementary table 


\section{TABLE 2 Baseline characteristics}

\begin{tabular}{|c|c|}
\hline Patients & 607 \\
\hline Age years & $65 \pm 9.2$ \\
\hline Female & 374 (62) \\
\hline \multicolumn{2}{|l|}{ Ethnicity } \\
\hline Caucasian & $506(84)$ \\
\hline African-American & $35(6)$ \\
\hline Asian & $37(6)$ \\
\hline Hispanic & $25(4)$ \\
\hline Native American & $2(<1)$ \\
\hline Clinical (c) tumour size $\mathrm{cm}$ & $1.94 \pm 0.77$ \\
\hline cT1a & $63(10)$ \\
\hline cT1b & $296(49)$ \\
\hline cT1c & $248(41)$ \\
\hline \multicolumn{2}{|l|}{ Tumour location } \\
\hline Right upper lobe & 197 (32) \\
\hline Right middle lobe & $59(10)$ \\
\hline Right lower lobe & 104 (17) \\
\hline Left upper lobe & $151(25)$ \\
\hline Left lower lobe & $96(16)$ \\
\hline \multicolumn{2}{|l|}{ Nodule density on CT } \\
\hline Solid & $400(66)$ \\
\hline Semisolid & $148(24)$ \\
\hline Ground glass & $59(10)$ \\
\hline EBUS-TBNA & $121(20)$ \\
\hline NO & 106 (17) \\
\hline N1 & $4(<1)$ \\
\hline N2 & $11(2)$ \\
\hline Lung resection & $596(98)$ \\
\hline Lobectomy & 454 (75) \\
\hline Segmentectomy & $83(14)$ \\
\hline Wedge resection & $76(12)$ \\
\hline Bilobectomy & $2(<1)$ \\
\hline Pneumonectomy & $1(<1)$ \\
\hline \multicolumn{2}{|l|}{ Histology } \\
\hline Adenocarcinoma & 441 (73) \\
\hline Squamous cell carcinoma & 77 (13) \\
\hline Neuroendocrine (carcinoid) & $76(13)$ \\
\hline Large cell carcinoma & $8(1)$ \\
\hline Adeno-squamous & $3(<1)$ \\
\hline Pleomorphic & $1(<1)$ \\
\hline NSCLC, NOS & $1(<1)$ \\
\hline \multicolumn{2}{|c|}{ Histological degree of differentiation } \\
\hline Well differentiated & $190(31)$ \\
\hline Moderately differentiated & 297 (49) \\
\hline Poorly differentiated & $96(16)$ \\
\hline Not available & $24(4)$ \\
\hline
\end{tabular}

Data are presented as $\mathrm{n}$, mean \pm SD or $\mathrm{n}(\%)$. CT: computed tomography; EBUS-TBNA: endobronchial ultrasound transbronchial needle aspiration; NSCLC: nonsmall cell lung cancer; NOS: not otherwise specified.

E6). Both definitions was tested in multivariate analysis and they remained statistically significant (supplementary table E7). We failed to demonstrate a difference in log odds ratio between Definitions 2 and 3.

\section{Discussion}

Our study suggests that in cT1N0M0 NSCLC the overall prevalence of occult "mediastinal" disease is relatively low regardless of tumour location. However, central location is associated with substantial risk of having occult nodal disease in any station $(\mathrm{pN} 1 / \mathrm{pN} 2 / \mathrm{pN} 3)$, which is of outmost importance for nonsurgical candidates with early lung cancer. This is the first study to objectively evaluate multiple definitions of tumour centrality with a dedicated software system. We identified two definitions associated with an increased probability of any occult nodal disease. Both entailed dividing the lungs into thirds 
TABLE 3 All patients: univariate logistic regression (pN0/pN1 versus pN2/pN3) \#

\begin{tabular}{|c|c|c|c|c|c|c|}
\hline Definition & Tumour location & Patients & Patients with pN2/pN3 & OR $(95 \% \mathrm{CI})$ & p-value & $\operatorname{AUC}(95 \% \mathrm{CI})$ \\
\hline 2 & Concentric inner two-thirds medial & 316 & $31(9.81)$ & $1.88(1.00-3.51)$ & 0.049 & $0.58(0.50-0.65)$ \\
\hline 4 & Concentric inner two-thirds centre & 237 & 18 (7.59) & $0.97(0.53-1.79)$ & 0.920 & $0.50(0.42-0.57)$ \\
\hline 5 & Vertical inner one-third medial & 123 & $12(9.76)$ & $1.39(0.70-2.77)$ & 0.348 & $0.53(0.46-0.59)$ \\
\hline 6 & Vertical inner two-thirds medial & 528 & $43(8.14)$ & $1.68(0.59-4.83)$ & 0.332 & $0.53(0.48-0.57)$ \\
\hline & Total & 607 & $47(7.73)$ & & & \\
\hline
\end{tabular}

Data are presented as $\mathrm{n}$ or $\mathrm{n}(\%)$, unless otherwise stated. AUC: area under the curve. ${ }^{\text {\# }}$ : modelled on probability of pN2/pN3.

TABLE 4 All patients: univariate logistic regression (pNO versus $\mathrm{pN} 1 / \mathrm{pN} 2 / \mathrm{pN} 3)^{\#}$

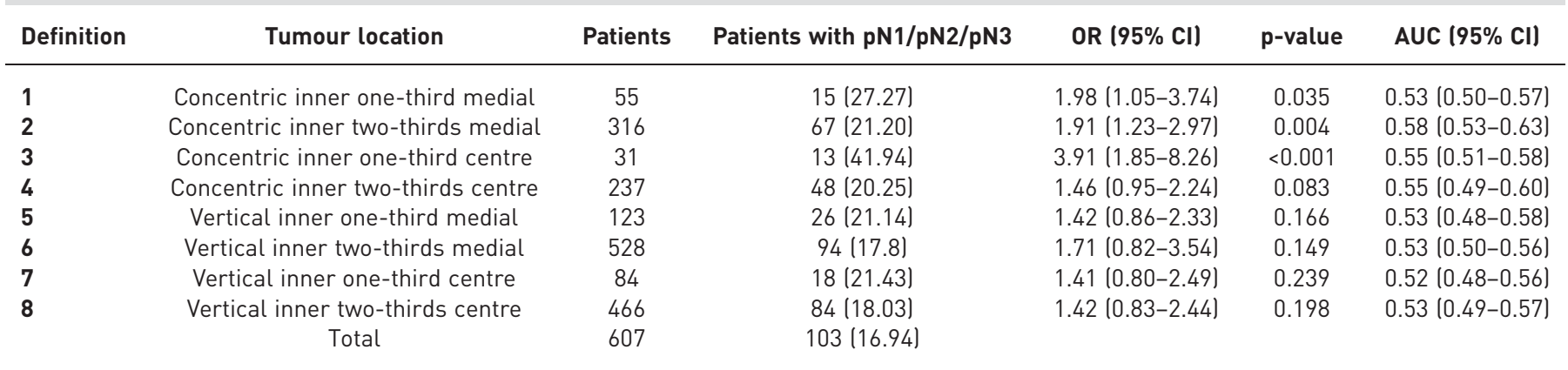

Data are presented as $\mathrm{n}$ or $\mathrm{n}(\%)$, unless otherwise stated. AUC: area under the curve. ${ }^{\text {\# }}$ : modelled on probability of pN1/pN2/pN3.

TABLE 5 Excluding patients without positron emission tomography-computed tomography: univariate logistic regression ( $\mathrm{pN} 0 / \mathrm{pN} 1$ versus $\mathrm{pN} 2 / \mathrm{pN} 3)^{\#}$

\begin{tabular}{|c|c|c|c|c|c|c|}
\hline Definition & Tumour location & Patients & Patients with pN2/pN3 & OR $(95 \% \mathrm{CI})$ & p-value & $\operatorname{AUC}(95 \% \mathrm{CI})$ \\
\hline 1 & Concentric inner one-third medial & 40 & $4(10.00)$ & $1.29(0.43-3.83)$ & 0.648 & $0.51(0.46-0.56)$ \\
\hline 2 & Concentric inner two-thirds medial & 257 & $26(10.12)$ & $1.83(0.91-3.65)$ & 0.088 & $0.57(0.49-0.65)$ \\
\hline 4 & Concentric inner two-thirds centre & 189 & 15 (7.94) & $0.96(0.49-1.89)$ & 0.912 & $0.50(0.41-0.58)$ \\
\hline 5 & Vertical inner one-third medial & 92 & 9 (9.78) & $1.30(0.59-2.84)$ & 0.514 & $0.52(0.45-0.59)$ \\
\hline 6 & Vertical inner two-thirds medial & 419 & 37 (8.83) & $2.91(0.68-12.37)$ & 0.149 & $0.54(0.50-0.58)$ \\
\hline 8 & Total & 481 & $39(8.11)$ & & & \\
\hline
\end{tabular}

Data are presented as $\mathrm{n}$ or $\mathrm{n}(\%)$, unless otherwise stated. AUC: area under the curve. ${ }^{\#}$ : modelled on probability of pN2/pN3.

following "concentric lines". One definition utilised the inner one-third of the lung (based on the centre of the tumour), whereas the other utilised the inner two-thirds of the lung (based on the most medial aspect of the tumour).

Accurate mediastinal staging is key in the management of lung cancer. In the context of patients with cT1N0M0 disease, current guidelines suggest that invasive mediastinal staging should be performed only for patients with "centrally located" tumours [1-3]. However, definitions of centrally located tumours are nonspecific, there is a large discrepancy among different guidelines and, as a result, there is heterogeneity in practice patterns [11]. Data supporting tumour centrality as a risk factor for occult mediastinal disease in lung cancer are scant, mostly retrospective and inconsistent [4-10]. A potential explanation for the inconsistency observed among the previous studies could be differences in the definitions of tumour 
TABLE 6 Excluding patients without positron emission tomography-computed tomography: univariate logistic regression (pNO versus $\mathrm{pN} 1 / \mathrm{pN} 2 / \mathrm{pN} 3)^{\#}$

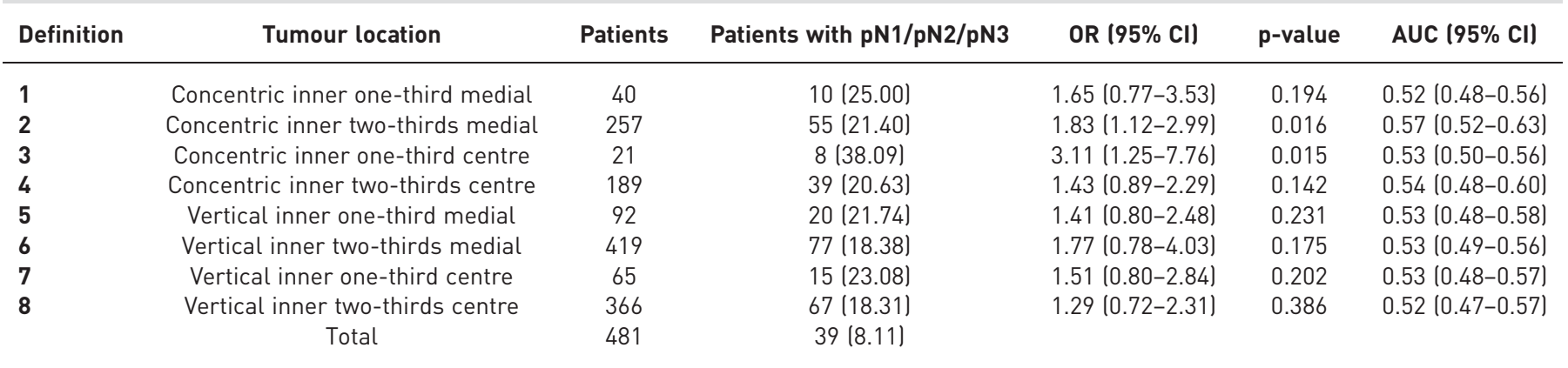

Data are presented as $\mathrm{n}$ or $\mathrm{n}(\%)$, unless otherwise stated. AUC: area under the curve. ${ }^{\text {\# }}$ : modelled on probability of pN1/pN2/pN3.

TABLE 7 Excluding patients with ground-glass opacities and carcinoids: univariate logistic regression (pNO versus $\mathrm{pN} 1 / \mathrm{pN} 2 / \mathrm{pN} 3)^{\#}$

\begin{tabular}{|c|c|c|c|c|c|c|}
\hline Definition & Tumour location & Patients & Patients with pN1/pN2/pN3 & OR $(95 \% \mathrm{CI})$ & p-value & $\operatorname{AUC}(95 \% \mathrm{CI})$ \\
\hline 1 & Concentric inner one-third medial & 39 & 13 (33.33) & $2.39(1.18-4.86)$ & 0.016 & $0.54(0.50-0.58)$ \\
\hline 2 & Concentric inner two-thirds medial & 236 & $56(23.73)$ & $1.96(1.22-3.13)$ & 0.005 & $0.58(0.53-0.64)$ \\
\hline 4 & Concentric inner two-thirds centre & 170 & $38(22.35)$ & $1.45(0.91-2.32)$ & 0.119 & $0.54(0.49-0.60)$ \\
\hline 5 & Vertical inner one-third medial & 99 & $24(24.24)$ & $1.55(0.91-2.63)$ & 0.107 & $0.54(0.49-0.59)$ \\
\hline 6 & Vertical inner two-thirds medial & 420 & 82 (19.52) & $1.70(0.78-3.70)$ & 0.183 & $0.53(0.49-0.56)$ \\
\hline 8 & Total & 484 & 90 (18.59) & & & \\
\hline
\end{tabular}

Data are presented as $\mathrm{n}$ or $\mathrm{n}(\%)$, unless otherwise stated. AUC: area under the curve. ${ }^{\#}$ : modelled on probability of pN1/pN2/pN3.

centrality as well as inconsistency in their application. Of note, each study evaluated a single definition of central tumours. In addition, most of these studies did not specify methods to delineate the three thirds of the lung and methods to classify a tumour that crosses a boundary (i.e. between a third that is considered central and one that is considered peripheral), introducing a large amount of subjectivity and bias. We have only identified one study that has evaluated different definitions of central tumours. In this recent study, DeCAluwé et al. [13] retrospectively studied 813 patients with cN0 disease (by CT and PET-CT) of which $42 \%$ were cT1, 28\% were cT2, 17\% cT3 and 11\% cT4. $97 \%$ of patients underwent surgery. They evaluated five definitions of tumour centrality: 1) inner one-third of the lung (measured by the location of the tumour centre, radially measured from the secondary carina on transverse CT image), 2) inner two-thirds (measured as the prior definition), 3) in contact with lobar or segmental branches of pulmonary vessels or bronchi, 4) within $2 \mathrm{~cm}$ of the central bronchial tree and 5) visualised during bronchoscopy. They studied the association of each of these definitions with the prevalence of $\mathrm{pN} 2 / \mathrm{pN} 3$ and that of $\mathrm{pN} 1 / \mathrm{pN} 2 / \mathrm{pN} 3$. Any nodal upstage ( $\mathrm{pN} 1 / \mathrm{pN} 2 / \mathrm{pN} 3)$ was found in $21 \%$ of the cases, but $\mathrm{pN} 2 /$ $\mathrm{pN} 3$ was found in only $8 \%$. Similarly to our study, they found no association between tumour centrality and occult $\mathrm{pN} 2 / \mathrm{pN} 3$ disease, but they did find association of tumour centrality with any pN upstage ( $\mathrm{pN} 1 / \mathrm{pN} 2 / \mathrm{pN} 3)$. However, unlike our study, all their five definitions of tumour centrality were associated with an increased odds of having any $\mathrm{pN}$ upstage $(\mathrm{pN} 1 / \mathrm{pN} 2 / \mathrm{pN} 3)$, with no particular definition having a stronger association. It is important to note that this study differs from ours in many ways, but particularly in their population and, of course, their methods. One of the most relevant differences in their population is that $58 \%$ of their patients had tumours $>3 \mathrm{~cm}$. We did not include these patients because invasive mediastinal staging would be already indicated based on tumour size (as indicated in all guidelines), making a classification of central versus peripheral less relevant. DeCALuwé et al. [13] do not report a secondary analysis including only patients with cT1 tumours, making a comparison with our study results more difficult. It is also not clear how many patients with tumours $>3 \mathrm{~cm}$ were in a central location for each of the five definitions, possibly introducing a bias. With regard to methods, in addition to testing different definitions, most of their CT scans were evaluated by a single reader, while we 
processed the CT scans with a dedicated software system to accurately determine the location of the tumour and to minimise subjectivity or human error.

In contrast to our study and that of DeCALUwé et al. [13], a study by GAO et al. [14] of risk factors for occult mediastinal disease in patients with early lung cancer staged with PET-CT reported a greater prevalence of occult $\mathrm{pN} 2$ disease in centrally located lesions. This was a retrospective study that included patients with $\mathrm{cT} 1 / \mathrm{cT} 2$, and utilised the centre of the tumour and the inner one-third of the lungs (measured as the radial distance from the hilum to the periphery of the lung) to define central tumours. The study does not report who reviewed the images or if there was a single or multiple chest CT reader(s). It included 165 patients with cT1 tumours, with 23 of those being central. The overall prevalence of occult $\mathrm{pN} 2$ disease in cT1 tumours was $3.6 \%$ and in cT2 tumours $11.8 \%$. The prevalence of occult pN2 disease for cT1 tumours was reported separately for solid tumours $(\mathrm{n}=71)$ and semisolid tumours $(\mathrm{n}=93)$. For solid cT1 tumours, it was $21.4 \%$ when central (three out of 24 ) versus $1.8 \%$ (one out of 57 ) when peripheral $(\mathrm{p}=0.022)$. For semisolid $\mathrm{cT} 1$ tumours, it was $11.1 \%$ when central (one out of nine) versus $1.2 \%$ (one out of 84 ) when peripheral $(\mathrm{p}=0.185)$. In our study, we combined solid and semisolid tumours, since the latter have a solid component that indicates invasiveness, and we only performed a separate analysis excluding purely ground-glass lesions, which are known to be less invasive.

It is important to be aware that there are other definitions of central/peripheral lung tumours that were created with different purposes, unrelated to the prevalence of occult mediastinal disease. The most popular definition is likely the one created by radiation oncologists, who define central tumours as those located within $2 \mathrm{~cm}$ of the proximal bronchial tree, heart, great vessels, trachea or other mediastinal structures $[15,16]$. This definition was developed to evaluate the safety of stereotactic body radiation therapy in central versus peripheral tumours and it is commonly used to adjust radiation doses. However, its ability to predict occult N2 disease was never tested and, hence, it should not be employed to decide whether or not invasive mediastinal staging is indicated.

One of the limitations of our study is that it was performed in a single institution. Our study population had a large proportion of patients with adenocarcinomas (73\%). Although we expected this characteristic of our population to increase the prevalence of occult nodal disease, the effect may have been mitigated by the significant prevalence of semisolid lesions and ground-glass opacities, which may, in fact, confer a protective effect (see table 2 and supplementary table E1a). Another limitation was the low prevalence of occult mediastinal disease in our population, which may have prevented us from demonstrating a significant difference between some of the proposed definitions of central tumours. Our inability to detect a difference among the definitions of tumour centrality that were associated with occult disease may, indeed, be due to a lack of power. In addition, the objective classification of tumours as central or peripheral provided by our dedicated software may not be reproducible when performed by physicians. Reproducibility of these definitions, and interobserver and intra-observer variability, need to be properly studied. Also, our process was not fully automated, since the centre or most medial aspect of each tumour was selected by the operator.

To the best of our knowledge, this is the largest study assessing multiple clear and specific definitions of tumour centrality for CT1N0M0 NSCLC and it is the only study to use dedicated software to facilitate this. Our data strongly support the use of concentric lines and not vertical lines to divide the lung. We believe that our results, along with the similar findings of DeCALUwé et al. [13] showing a low prevalence of N2 disease regardless of tumour location, may help inform the current guidelines [13]. These findings may challenge the recommendation for invasive mediastinal staging in patients with central cT1 tumours, particularly when surgery is the planned therapy. The radiographic group " $\mathrm{C}$ " from the American College of Chest Physicians guidelines that combines patients with $\mathrm{cN} 1$ disease with those with central tumours may need to be revised, since these may be two different kinds of patients with dissimilar risk of occult N2 disease [1]. Nevertheless, tumour centrality should remain an indication for systematic invasive nodal staging (mediastinal and hilar) for patients with cT1N0M0 tumours who will undergo a nonsurgical local ablative treatment.

\section{Conclusions}

Our study found the overall prevalence of occult "mediastinal" disease to be relatively low regardless of tumour location in CT1N0M0 NSCLC. However, central location was associated with substantial risk of having occult nodal disease in any station $(\mathrm{N} 1 / \mathrm{N} 2 / \mathrm{N} 3)$, which is of outmost importance for nonsurgical candidates with early lung cancer. We identified two definitions of central tumours and both entailed the use of concentric lines (and not vertical lines) to divide the lungs in thirds.

Author contributions: R.F. Casal contributed to the study design, institutional review board application, software design, image processing, data collection and interpretation, and manuscript composition and revision. He had full access to all 
the data in the study, and takes responsibility for the integrity of the data and the accuracy of the data analysis. B. Sepesi contributed to study design, data interpretation, and manuscript composition and revision. J. Tschirren contributed to software design and manuscript revision. M. Chen and L. Li contributed to study design, statistical analysis, data interpretation, and manuscript composition and revision. A-E.S. Sagar, J. Sunny and J. Williams contributed to data collection and interpretation, and manuscript composition and revision. H.B. Grosu, G.A. Eapen and C.A. Jiminez contributed to data interpretation, and manuscript composition and revision. D.E. Ost contributed to study design, data interpretation, and manuscript composition and revision.

Conflict of interest: R.F. Casal reports grants from Siemens and Concordia, and personal fees for consultancy from Olympus and Boston Scientific, outside the submitted work. B. Sepesi has nothing to disclose. A-E.S. Sagar has nothing to disclose. J. Tschirren has nothing to disclose. M. Chen has nothing to disclose. L. Li has nothing to disclose. J. Sunny has nothing to disclose. J. Williams has nothing to disclose. H.B. Grosu has nothing to disclose. G.A. Eapen reports grants from Spiration, outside the submitted work. C.A. Jimenez reports grants from Care Fusion, outside the submitted work. D.E. Ost has nothing to disclose.

Support statement: Imaging software was provided by VIDA Diagnostics (Coralville, IA, USA).

\section{References}

1 Silvestri GA, Gonzalez AV, Jantz MA, et al. Methods for staging non-small cell lung cancer: diagnosis and management of lung cancer, 3rd ed: American College of Chest Physicians evidence-based clinical practice guidelines. Chest 2013; 143: 5 Suppl., e211S-e250S.

2 De Leyn P, Dooms C, Kuzdzal J, et al. Preoperative mediastinal lymph node staging for non-small cell lung cancer: 2014 update of the 2007 ESTS guidelines. Transl Lung Cancer Res 2014; 3: 225-233.

3 Ettinger DS, Akerley W, Borghaei H, et al. Non-small cell lung cancer, version 2.2013. J Natl Compr Canc Netw 2013; 11: 645-653.

4 Ketchedjian A, Daly BD, Fernando HC, et al. Location as an important predictor of lymph node involvement for pulmonary adenocarcinoma. J Thorac Cardiovasc Surg 2006; 132: 544-548.

5 Lee PC, Port JL, Korst RJ, et al. Risk factors for occult mediastinal metastases in clinical stage I non-small cell lung cancer. Ann Thorac Surg 2007; 84: 177-181.

6 Gomez-Caro A, Garcia S, Reguart N, et al. Incidence of occult mediastinal node involvement in $\mathrm{cN} 0$ non-small-cell lung cancer patients after negative uptake of positron emission tomography/computer tomography scan. Eur J Cardiothorac Surg 2010; 37: 1168-1174.

7 Kanzaki R, Higashiyama M, Fujiwara A, et al. Occult mediastinal lymph node metastasis in NSCLC patients diagnosed as clinical N0-1 by preoperative integrated FDG-PET/CT and CT: risk factors, pattern, and histopathological study. Lung Cancer 2011; 71: 333-337.

8 Chen K, Yang F, Jiang G, et al. Development and validation of a clinical prediction model for N2 lymph node metastasis in non-small cell lung cancer. Ann Thorac Surg 2013; 96: 1761-1768.

9 Zhang Y, Sun Y, Xiang J, et al. A prediction model for N2 disease in T1 non-small cell lung cancer. J Thorac Cardiovasc Surg 2012; 144: 1360-1364.

10 Farjah F, Lou F, Sima C, et al. A prediction model for pathologic N2 disease in lung cancer patients with a negative mediastinum by positron emission tomography. J Thorac Oncol 2013; 8: 1170-1180.

11 Casal RF, Vial MR, Miller R, et al. What exactly is a centrally located lung tumor? Results of an online survey. Ann Am Thorac Soc 2017; 14: 118-123.

12 Rami-Porta R, Bolejack V, Crowley J, et al. The IASLC Lung Cancer Staging Project: proposals for the revisions of the T descriptors in the forthcoming eighth edition of the TNM classification for lung cancer. $J$ Thorac Oncol 2015; 10: 990-1003.

13 Decaluwé $\mathrm{H}$, Moons J, Fieuws S, et al. Is central lung tumour location really predictive for occult mediastinal nodal disease in (suspected) non-small-cell lung cancer staged $\mathrm{cN} 0$ on ${ }^{18} \mathrm{~F}$-fluorodeoxyglucose positron emission tomography-computed tomography? Eur J Cardiothorac Surg 2018; 54: 134-140.

14 Gao SJ, Kim AW, Puchalski JT, et al. Indications for invasive mediastinal staging in patients with early non-small cell lung cancer staged with PET-CT. Lung Cancer 2017; 109: 36-41.

15 Park HS, Harder EM, Mancini BR, et al. Central versus peripheral tumor location: influence on survival, local control, and toxicity following stereotactic body radiotherapy for primary non-small-cell lung cancer. $J$ Thorac Oncol 2015; 10: 832-837.

16 Timmerman R, Paulus R, Galvin J, et al. Stereotactic body radiation therapy for inoperable early stage lung cancer. JAMA 2010; 303: 1070-1076. 